\title{
Current Status of Nanoparticles Loaded Medication in the Management of Diabetic Retinopathy
}

\author{
Dhanraj Ganapathy ${ }^{1}$, Rajeshkumar Shanmugam², Durairaj Sekar³
}

\begin{abstract}
${ }^{1}$ Department of Prosthodontics, Saveetha Dental College and Hospitals, Saveetha Institute of Medical and Technical Sciences, Chennai, Tamilnadu, India. ${ }^{2}$ Department of Pharmacology, Saveetha Dental College and Hospitals, Saveetha Institute of Medical and Technical Sciences, Chennai, Tamilnadu, India. ${ }^{3}$ Department of Pharmacology, Saveetha Dental College and Hospitals, Saveetha Institute of Medical and Technical Sciences, Chennai, Tamilnadu, India.
\end{abstract}

\section{ABSTRACT}

\section{BACKGROUND}

Diabetes Mellitus (DM) is a chronic metabolic disorder affecting a vast majority of the human population causing marked morbidity and mortality. Diabetic retinopathy (DR) is a complication of diabetes that affects the eyes and vision. It is a leading cause of visual impairment and blindness in people. In this review, various nano-delivery systems to effectively deliver antidiabetic compounds in treating angiopathy and retinopathy in diabetes mellitus are discussed. This has been used to overcome many complications in traditional treatment of bioactive compounds with a lower potential antidiabetic effect due to the lower stability of those compounds in gastrointestinal systems and during absorption. Several bioactive compounds loaded into nanodelivery systems are currently in clinical trials, and once these compounds are commercially marketed, nano bioactive compounds will be available as novel medicines to treat many chronic diseases, including diabetes mellitus. DR is driven by prolonged hyperglycaemic episodes arising from suboptimal glycaemic control in patients with either Type I or II diabetes mellitus. Elevated blood glucose levels alter the regulation of a number of biochemical pathways leading to superoxide production and oxidative stress in retina. Mitochondrial dysfunction, inflammation, and hypoxia-driven Vascular Endothelial Growth Factor (VEGF) secretion giving rise to vascular and neuronal apoptosis, neovascularization and elevated vasopermeability, respectively. Several medicines loaded with nanoparticles have been developed to enhance the target effectiveness and bioavailability of medicinal compounds with antidiabetic effects in various animal and human models. Bioactive compounds have been loaded into nanoparticles for oral delivery in various antidiabetic animal models, and the results have shown improved stability, bioavailability, and sustained antidiabetic effects. Nanocarriers used in the delivery of drugs are very precise and ensure targeted drug delivery at the disease site. The antidiabetic activity of triamcinolone acetonide-loaded lipid nanocapsules, humanin peptide with elastin like polypeptide nanoassembly, loaded poly lactic-co-glycolic acid nanoparticles, anti-VEGF-aptamer modified C-Dots as hybrid nano-composite, axitinib, apatinib loaded poly lactic-coglycolic acid nanoparticles, mulberry leaf extract mediated silver nanoparticles, resveratrol coated gold nanoparticles, fenofibrate-loaded biodegradable nanoparticles, nano-delivery of doxorubicin, insulin-loaded chitosan nanoparticles, silk fibroin nanoparticles, fluorescent silicon nanoparticles-based theranostic probes, topical curcumin nanocarriers, nanoparticles loaded-palmitoyl ethanolamide, hyaluronic acid coated albumin nanoparticles, nilvadipine loaded nanoparticles, cilostazol ophthalmic nanodispersions, naringenin-loaded sulphobutylether- $\beta$-cyclodextrin/chitosan nanoparticles, emodin-loaded magnesium silicate hollow nanocarriers, yttrium oxide nanoparticles are discussed in this review.

\section{KEY WORDS}

Diabetes Mellitus, Diabetic Retinopathy, Angiopathy, Nano Particles, Ocular Disorders
Corresponding Author: Dr. Rajeshkumar Shanmugam, Associate Professor, Nanobiomedicine Lab, Department of Pharmacology, Saveetha Dental College and Hospitals, Saveetha Institute of Medical \&

Technical Sciences, Chennai-600077, Tamilnadu, India.

E-mail: ssrajeshkumar@hotmail.com

DOI: $10.14260 /$ jemds/2020/376

Financial or Other Competing Interests: None.

How to Cite This Article: Ganapathy D, Shanmugam $R$, Sekar D. Current status of nanoparticles loaded medication in the management of diabetic retinopathy. J. Evolution Med. Dent. Sci. 2020;9(22):1713-1718, $10.14260 /$ jemds $/ 2020 / 376$

Submission 04-03-2020, Peer Review 07-05-2020, Acceptance 16-05-2020, Published 01-06-2020.

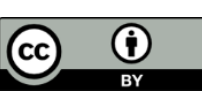




\section{BACKGROUND}

Diabetes Mellitus is a chronic metabolic disorder affecting a vast majority of human population causing marked morbidity and mortality.[1,2] This disorder is associated with several etiologic factors including genetics, age, life style, lack of physical activity, dietary habits, high physical and mental stress, inflammation, and obesity. Diabetic retinopathy (DR) is a complication of diabetes that affects the eyes and vision. It is a leading cause of visual impairment and blindness in people. ${ }^{[3]}$ Vascular endothelial growth factor-A (VEGF-A) is a primary initiator and potential mediator of DR. ${ }^{[4]}$

DR is driven by prolonged hyperglycaemic episodes arising from suboptimal glycaemic control in patients with either type I or II diabetes mellitus (DM) Elevated blood glucose levels alter the regulation of a number of biochemical pathways leading to superoxide production and oxidative stress in retina. Mitochondrial dysfunction, inflammation, and hypoxia-driven vascular endothelial growth factor (VEGF) secretion give rise to vascular and neuronal apoptosis, and neovascularization and elevated vasopermeability, respectively.[2-4] Several medicines are loaded with nanoparticles have been developed to enhance the target effectiveness and bioavailability of medicinal compounds with antidiabetic effects in various animal and human models.[5] Bioactive compounds have been loaded into nanoparticles for oral delivery in various antidiabetic animal models, and the results have shown improved stability, bioavailability, and sustained antidiabetic effects.[6,7] The nanocarriers used in the delivery of drugs are very precise and ensure targeted drug delivery at the disease site. ${ }^{[8]}$ The currently used formulations in the management of retinopathy are as follows.

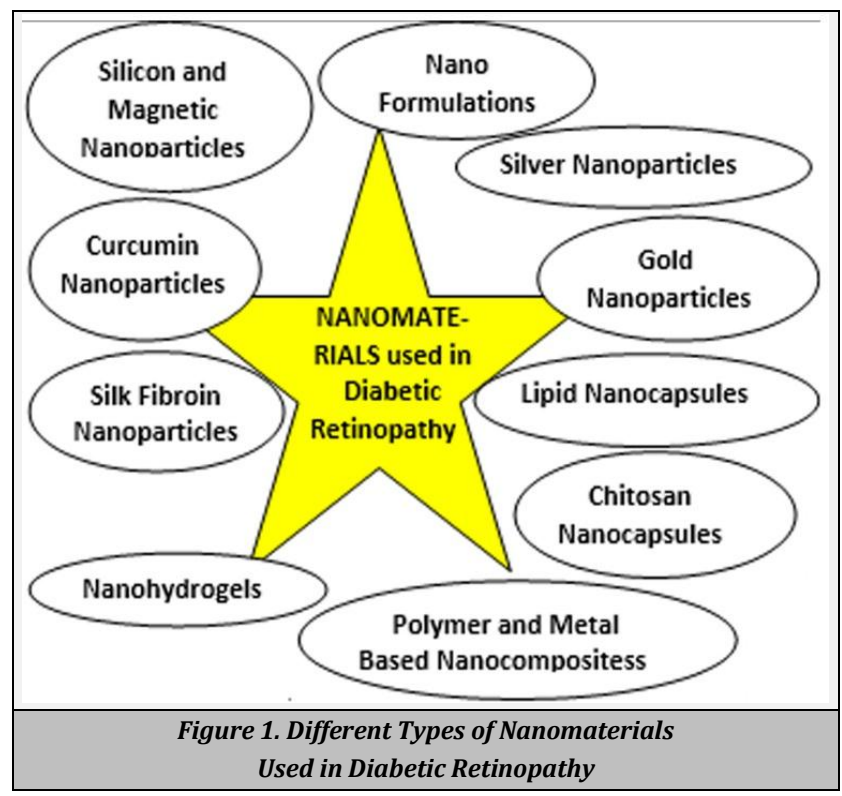

Triamcinolone Acetonide-Loaded Lipid Nanocapsules[ ${ }^{[9]}$ Triamcinolone acetonide (TA) was formulated with nano capsules and used as an effective vehicle to ensure more precise targeted drug delivery in angiopathic and inflammatory ocular disorders. A high anti-inflammatory activity below the toxicity level, was shown by Triamcinolone acetonide lipid nanocapsules with a reduction of $30 \%$ in interleukin 6 secretion observed in vitro models. The TALNCs also revealed a therapeutic efficacy in the endotoxininduced uveitis in rabbit model with a significant attenuation of clinical signs of an inflammatory response.

\section{Humanin Peptide with Elastin Like Polypeptide Nanoassembly[4]}

Humanin (HN) is a hydrophobic 24-amino acid peptide derived from mitochondrial DNA that regulate and modulate cellr responses to oxidative stress and protects human retinal pigment epithelial (RPE) cells from apoptosis. Li Z et al[10] reported two genetically encoded fusions between $\mathrm{HN}$ and elastin-like polypeptides (ELP) to solubilize Humanin. ELPs provide steric stabilization and thermo-responsive phase separation. Both fusions bind human RPE cells and protect against oxidative stress-induction of apoptosis. Human inpolypeptides may regulate the cellular delivery of biodegradable nanoparticles. They offer potential protection against all age-related disorders, including diabetic retinopathy and macular degeneration.

Loaded Poly (Lactic-Co-Glycolic Acid) Nanoparticles [11,12] Interleukin-12 (IL-12) is a cytokine of the chemokine family that could reduce the levels of MMP-9 and VEGF-A and suppress tumor angiogenesis. IL-12 can also have superior therapeutic effectiveness against Diabetic retinopathy. IL-12loaded poly (lactic-co-glycolic acid) nanoparticles can facilitate long-term and sustained DR treatment. IL-12-PNP demonstrated better inhibitory efficacy against VEGF-A and MMP-9 expression in rat endothelial cells and DR mouse retina. Intraocular IL-12-PNP administration significantly reduced retinal damage in DR mice as they presented with increased thickness and decreased neovascularization after treatment.

In the study by Zhang XP et al,[13] bevacizumab was encapsulated into poly (lactide-co-glycolide) (PLGA) nanoparticles. PLGA encapsulation could prolong the residency of bevacizumab in the vitreous and aqueous humor and produce long-lasting drug concentrations. Bevacizumabencapsulated PLGA had no significant cytotoxicity and tissue toxicity effect in vitro and in vivo. Bevacizumab-encapsulated PLGA was more effective than bevacizumab in inhibiting VEGF-mediated endothelial cell proliferation, migration and tube formation. Bevacizumab-encapsulated PLGA enhanced the anti-angiogenic efficiency of bevacizumab for treating corneal neovascularization and retinal neovascularization.

\footnotetext{
Anti-VEGF-Aptamer Modified C-Dots as Hybrid Nanocomposite

Carbon dots (C-dots) functionalized with the VEGF aptamer was introduced by Shoval $\mathrm{A}$ et al,[14] and the hybrid nanoparticles were used for ocular nanomedicine. The Carbon-dots were applied as effective carriers of the antiVEGF aptamer across the cornea, yielding therapeutic levels upon topical administration. The hybrid aptamer-modified Cdots can provide a versatile nanomedicine to treat agerelated macular degeneration and diabetic retinopathy.
} 


\section{Axitinib, Apatinib Loaded Poly (Lactic-Co-Glycolic Acid) Nanoparticles}

Axitinib, multireceptor tyrosine kinase inhibitor, is a small molecule that works by blocking vascular endothelial growth factor receptors (VEGFR) and platelet-derived growth factor receptors (PDGFR) responsible for developing neovascularization. Narvekar $P$ et al,[15] developed a sustained release formulation of axitinib-loaded poly (lacticco-glycolic acid) (PLGA) nanoparticles to minimize frequent administration of the drug by intravitreal injection. Cellular uptake, antimigration assay, and VEGF expression levels for the nanoparticles suggested greater uptake, significant antiangiogenic potential, and inhibition of VEGF activity and hence can be used in the management of Diabetic Retinopathy.

Halasz $\mathrm{K}$ et $\mathrm{al}^{[16]}$ showed that apatinib nano particles decreased toxicity (1.36 \pm 0.74 fold) and efficient VEGF inhibition ( $3.51 \pm 0.02$ fold) via VEGFR2 mediation and showed potential to be developed as medication in the treatment of Diabetic Retinopathy. Jeong JH et al[17] investigated whether apatinib-loaded polymeric nanoparticles inhibit VEGF-mediated retinal vascular hyperpermeability and block diabetes-induced retinal vascular leakage. The data indicated that Apa-HSA-PEG nano particles efficiently blocked VEGF-induced breakdown of the bloodretinal barrier. In vivo experiments with streptozotocininduced diabetic mice showed that an intravitreal injection of Apa-HSA-PEG nanoparticles substantially inhibited diabetesinduced retinal vascular leakage.

\section{Mulberry Leaf Extract Mediated Silver Nanoparticles}

$\mathrm{Xu} \mathrm{L}$ et al[18] used Mulberry leaf extract biomolecules as capping and reducing agent for fabrication of Silver nanoparticles AgNPs in the management of diabetic retinopathy in rats. Later, the fabricated AgNPs were characterized by using spectroscopic and microscopic instrumental techniques such as HR-TEM, UV-Vis, XRD and FT-IR. EDS, XRD and TEM have confirmed the synthesis of AgNPs. HRTEM results exhibited that the formed AgNPs are polydispersed and spherical in nature with mean particle size of $35 \mathrm{~nm}$. Microscopic observation in retina of diabetic rats showed noticeable amelioration and therapeutic activity against diabetic retinopathy.

\section{Resveratrol Coated Gold Nanoparticles[19]}

Gold nanoparticles (AuNPs) were coated with plant polyphenol called Resveratrol which acted acted as both stabilizing and reducing agent. The prepared AuNPs were tested on streptozotocin (STZ) induced diabetic rats for their amelioration consequence. The retinal vessels in the AuNPs administrated diabetic rats were observed to be decreased through the retinal histopathological examination. In the AuNPs administrated diabetic rats, the retinal expression of renal Pigment Epithelium-Derived Factor (PEDF) was observed to be increased and the Vascular Endothelial Growth Factor (VEGF-1), which was increased in diabetic rats was declined on treating with AuNPs. On treating the STZ injected diabetic rats with AuNPs, all the retinal mRNA expressions of VEGF-1, Tumour Necrosis Factor (TNF $\alpha$ ),
Monocyte Chemotactic Proteins-1 (MCP-1), Intercellular Adhesion Molecule-1 (ICAM-1), and Interleukin (IL)-6, IL-1 $\beta$ were observed to be reduced. Furthermore, AuNPs can reduce phosphorylation of Nuclear Factor Kappa B (NF-kB) p65 and Extracellular signal Regulated Kinase (ERK) 1/2 along with a growth in nuclear translocation of pNF- $\kappa \mathrm{B}$ p65 produced by STZ.

\section{Fenofibrate-Loaded Biodegradable Nanoparticles ${ }^{[20]}$}

Fenofibrate is a peroxisome proliferator-activated receptor $\alpha$ (PPAR $\alpha$ ) agonist and has been shown to have therapeutic effects on diabetic retinopathy (DR). However, the effects of fenofibrate through systemic administration are not as potent as desired due to inefficient drug delivery to the retina. Qiu F et al[20] explored the sustained therapeutic effects of fenofibrate-loaded bio-degradable nanoparticles (NP) on both DR and neovascular age-related macular degeneration (AMD). In diabetic rats, Feno-NP ameliorated retinal dysfunctions, reduced retinal vascular leakage, inhibited retinal leukostasis, and down regulated the overexpression of VEGF and ICAM-1 at 8 weeks after one IVT injection. In addition, Feno-NP reduced retinal vascular leakage and CNV formation in both CNV rats and Vldlr-/-mice. Moreover, no toxicity of Feno-NP or Blank-NP to retinal structure and function was detected. Feno-NP exhibited good physiochemical characteristics and controlled drug release profile, conferring prolonged beneficial effects on DR and neovascular AMD.

\section{Nano Delivery of Doxorubicin[21]}

Polymeric nanoparticles (NPs) containing doxorubicin (DOX) were prepared for the inhibition of hypoxia-induced factor $1 \alpha$ (HIF-1 $\alpha$ ). HIF-1 $\alpha$ is responsible for the upregulation of several angiogenic factors, including vascular endothelial growth factor (VEGF). DOX inhibits HIF- $1 \alpha$ but is highly toxic. By encapsulating DOX in NPs, drug delivery will be sustained and toxicity will be reduced without limiting efficacy.

DOX NPs were prepared using both poly lactic-co-glycolic acid (PLGA) and chitosan. PLGA NPs were prepared via nanoprecipitation (NPC) and single and double emulsion diffusion (SE; DE). The NP formulations decreased HIF-1 $\alpha$ and VEGF-A expression in ARPE-19 cells with reduced cytotoxicity. SE, DE, and CC demonstrated low ZP as well as the most rapid drug release of the tested formulations. FTIR confirmed the presence of DOX on the SE NP surface, indicating instability NPC was the most efficient formulation for the nanodelivery of DOX for AMD.

\section{Insulin-Loaded Chitosan Nanoparticles/PLGA-PEG-PLGA Hydrogel[22]}

Rong X et al[22] developed a novel insulin delivering system by loading onto chitosan nanoparticles/poly (lactic-co-glycolic acid)-poly (ethylene glycol)-poly (lactic-co-glycolic acid) hydrogel (ICNPH) for sustained release systems for insulin to treat diabetic retinopathy (DR) and reported effective results. Examinations including electroretinography, HE staining, transmission electron microscopy, terminal deoxynucleotidyl 
transferase d UTP nick-end labeling, immunofluorescence, Western blot, and real-time polymerase chain reaction were performed to evaluate the neuroprotective efficacy of ICNPH on DR by a single subconjunctival injection. ICNPH demonstrated sufficient neuroprotective effect on retinas through subconjunctival injection in DR rats and facilitates controlled insulin delivery.

\section{Silk Fibroin Nanoparticles[23]}

SFNs silk fibroin nanoparticles were prepared with regenerated silk fibroin using desolvation method with fluorescein isothiocyanate labelled bovine serum albumin (FITC-BSA) as bio-macromolecular model drug encapsulated. In vitro physicochemical properties and in vitro drug release of FITC-BSA loaded SFNs (FITC-BSA-SFNs) were evaluated. FITC-BSA-SFNs achieved accumulated distribution and extended retention in retina in vivo following intravitreal injection compared to a single administration of free drug solution. Therefore, this bio-macromolecule delivery platform based on SFNs could have great potential in the treatment of posterior segment and angiopathic disorders.

\section{Topical Curcumin Nanocarriers ${ }^{24]}$}

Curcumin (1,7-bis-(4-hydroxy-3-methoxyphenyl) -1,6heptadiene- 3,5 dione) is a polyphenol extracted from turmeric that has long been advocated for the treatment of a variety of conditions including neurodegenerative and inflammatory disorders. Davis BM et al[24] described a novel nanocarrier formulation comprising Pluronic-F127 stabilised D- $\alpha$-Tocopherol polyethene glycol 1000 succinate nano particles, which were used to successfully solubilize high concentrations $(4.3 \mathrm{mg} / \mathrm{mL})$ of curcumin. Curcumin-loaded nano carriers (CN) were found to significantly protect against cobalt chloride induced hypoxia and glutamate induced toxicity in vitro, with $\mathrm{CN}$ treatment significantly increasing R28 cell viability. Using established glaucoma-related in vivo models of ocular hypertension (OHT) and partial optic nerve transection (pONT), topical application of $\mathrm{CN}$ twice-daily for three weeks significantly reduced retinal ganglion cell loss compared to controls. These results suggest that novel topical $\mathrm{CN}$ formulation has potential as an effective neuro protective therapy in glaucoma and other eye diseases with neuronal and angiopathic pathology.

\section{Fluorescent Silicon Nanoparticles-Based Theranostic Probes}

Tang $\mathrm{M}$ et al ${ }^{[25]}$ presented a kind of theranostic agents made of peptide-functionalized silicon nanoparticles (SiNPs), suitable for simultaneous ocular neovascularization imaging and therapy. SiNPs-RGD can be used for labelling angiogenic blood vessels and neovascularization suppression, demonstrating obvious inhibition of new blood vessels formation in mouse corneas. The results suggest the SiNPsRGD as a novel class of high-quality theranostic probes is suitable for simultaneous diagnosis and treatment in ocular neovascular diseases.

\section{Ranibizumab Fabricated PLGA-PEGylated Magnetic Nanoparticles}

Ranibizumab is an active humanized monoclonal antibody that counteracts active forms of vascular endothelial growth factor A in the neovascular age-related macular degeneration therapy. The development of ranibizumab-related therapies, Yan J et al[26] designed the effective drug career with engineered magnetic nanoparticles $\left(\mathrm{Fe}_{3} \mathrm{O}_{4}\right)$ as a facile platform of ranibizumab delivery for the treatment of neovascular age-related macular degeneration. Ranibizumab conjugated $\mathrm{Fe}_{3} \mathrm{O}_{4} /$ PEG-PLGA polymer nanomaterials can be highly favourable and novel formulation for the treatment of neovascular age-related macular degeneration.

\section{Nanoparticles Loaded with Palmitoylethanolamide[27]}

Nanostructured lipid carriers (NLCs) loaded with palmitoylethanolamide (PEA) were formulated to enhance ocular bioavailability of PEA, particularly to the posterior portion of the eye. The novel formulation significantly increased PEA levels in ocular tissues compared to PEA suspension. The PEA-NLC formulation was characterized by high stability and robust ocular bioavailability. Therefore, this innovative formulation may be useful in clinical practice to manage retinal diseases.

\section{Hyaluronic Acid Coated Albumin Nanoparticles[28]}

Huang D et al[28] aimed to achieve targeted and sustained peptide delivery to the retina by encapsulating $\mathrm{Cx} 43$ MP into hyaluronic acid (HA) coated albumin nanoparticles(NPs). Intraocular biodistribution, particle retention, retinal targeting, and therapeutic efficacy of intravitreally injected NPs encapsulating Cx43 MP were evaluated in a rat model of retinal ischaemia-reperfusion injury. NPs rapidly diffused through the vitreous and specifically targeted CD44 expressing retinal cells. NPs remained at the target site for extended periods enabling sustained peptide release and thus prolonged therapeutic action.

\section{Nilvadipine Loaded Nanoparticles ${ }^{[29]}$}

Deguchi $S$ et al ${ }^{[29]}$ prepared ophthalmic formulations containing nilvadipine nanoparticles (NILnano) and demonstrated the instillation of NILnano can prevent retinal dysfunction in rats injected with excessive streptozotocin (STZ rats). NILnano(mean particle size, $77 \mathrm{~nm}$ ) was prepared by wet bead mill treatment, with the inclusion of various additives (2-hydroxypropyl- $\beta$-cyclodextrin, benzalkonium chloride, d-mannitol, and methylcellulose. The instillation of NILnano allowed the topical supplement of nilvadipine into the retina, and repeated instillation of NILnano (2 times/day) attenuated the retinal disorders led by the excessive streptozotocin.

\section{Cilostazol Ophthalmic Nanodispersions ${ }^{[30]}$}

Nagai $\mathrm{N}$ et al[30]prepared ophthalmic formulations containing cilostazol (CLZ) nanoparticles by bead mill methods (CLZnano) and found that instillation of CLZnano into rat eyes supplies CLZ into the retina. They demonstrated that dispersions containing CLZ nanoparticles attenuate changes in the ERG of 
STZ rats. In addition, the level of vascular endothelial growth factor (VEGF) in the retinas of STZ rats was 9.26-fold higher than in in normal rats, with this increase also prevented by the instillation of CLZnano

Wang $J$ et al[31] showed Chit-DC-VB12 nanoparticles loaded with scutellarin have better bioavailability and cellular uptake efficiency than Scutellarin, while Chit-DCVB12-Scunano particles alleviated the structural disorder of intraretinal neovessels in the retina induced by diabetes, and it also inhibited the retinal neovascularization via downregulated the expression of angiogenesis proteins. The ChitDC-VB12 nanoparticles enhanced scutellarin oral delivery efficacy and exhibited potential as small intestinal target promising nano-carriers for treatment of type II diabetes induced-retinopathy.

\section{Nanoparticle-Mediated miR200-b}

Mitra RN et al[32] investigated the effect of miR200-b, a potential anti-angiogenic factor, on VEGF receptor 2 (VEGFR2) expression and to determine the underlying angiogenic response in mouse endothelial cells, and in retinas from aged Ins2 (Akita) mice. Intravitreal injection of miR200-b DNA NPs significantly reduced protein levels of VEGFR-2 as revealed by western blot and markedly suppressed angiogenesis as evaluated by fundus imaging in aged Ins2 (Akita) mice even after 3 months of post-injection. These findings suggest that NP-mediated miR200-b delivery has negatively regulated VEGFR-2 expression in vivo.

\section{Naringenin Loaded Sulphobutylether- $\beta$ - Cyclodextrin/Chitosan Nanoparticles \\ Zhang $\mathrm{P}$ et al[33] prepared and characterized the naringenin- loaded sulphobutylether- $\beta$-cyclodextrin/chitosan nanoparticles (Nag-CD/CS-NPs) and evaluate their potential for the topical ophthalmic delivery. The study indicated that Nag-CD/CS-NPs achieved moderate sustained-release effect, and the in vivo study revealed that the prepared nano particles was nonirritating to rabbit's eye and had better ability to prolong the residence time than the naringenin suspension, which can significantly increase naringenin bioavailability in the aqueous humor.}

\footnotetext{
Emodin-Loaded Magnesium Silicate Hollow Nanocarriers ${ }^{[34]}$

Magnesium silicate hollow spheres were synthesized, characterized and cytotoxicity was evaluated in retina capillary endothelial cells. The loading and releasing capacity were tested by employing emodin, and the effect on VEGF expression was performed at the gene and protein level. Finally, an investigation on angiogenesis was carried on fertilized chicken eggs. The results indicated that the magnesium silicate nano particles had low toxicity. Emodin$\mathrm{MgSiO}_{3}$ can inhibit the expression of both VEGF gene and protein effectively. Angiogenesis of eggs was also reduced significantly.
}

\section{Yttrium Oxide Nanoparticles[35]}

Yttrium oxide $\left(\mathrm{Y}_{2} \mathrm{O}_{3}\right)$ nanoparticles (NPs) are excellent free radical scavengers owing to their nonstoichiometric crystal defects. A murine light-stress model was used to test the efficacy of $\mathrm{Y}_{2} \mathrm{O}_{3} \mathrm{NPs}(\sim 10-14 \mathrm{~nm}$ in diameter) in ameliorating retinal oxidative stress-associated degeneration. Intravitreal injections of these NPs at doses ranging from 0.1 to $5.0 \mu \mathrm{M} 2$ weeks before acute light stress protect PRs from degeneration. $\mathrm{Y}_{2} \mathrm{O}_{3}$ NPs were nontoxic and well tolerated after intravitreal delivery.

\section{CONCLUSIONS}

In this review, we have discussed various nanodelivery systems to effectively deliver antidiabetic compounds in treating retinopathy in diabetes mellitus. This could be used to overcome many complications in traditional treatment of bioactive compounds with a lower potential antidiabetic effect due to the lower stability of those compounds in gastrointestinal systems and during absorption. Several bioactive compounds loaded into nanodelivery systems are currently in clinical trials, and once these compounds are commercially marketed, nano bioactive compounds will be available as novel medicines to treat diabetic retinopathy.

\section{REFERENCES}

[1] Whiting DR, Guariguata L, Weil C, et al. IDF diabetes atlas: global estimates of the prevalence of diabetes for 2011 and 2030. Diabetes Research and Clinical Practice 2011;94(3):311-21.

[2] Wu YL, Ding YP, Tanaka Y, et al. Risk factors contributing to type 2 diabetes and recent advances in the treatment and prevention. Int J Med Sci 2014;11(11):1185-200.

[3] Thomas RL, Halim S, Gurudas S, et al. IDF Diabetes Atlas: a review of studies utilising retinal photography on the global prevalence of diabetes related retinopathy between 2015 and 2018. Diabetes Research and Clinical Practice 2019;157:107840.

[4] Chen HJ, Ma ZZ, Li Y, et al. Change of vascular endothelial growth factor levels following vitrectomy in eyes with proliferative diabetic retinopathy. Journal of Ophthalmology 2019;2019:6764932.

[5] Whitehead M, Wickremasinghe S, Osborne A, et al. Diabetic retinopathy: a complex pathophysiology requiring novel therapeutic strategies. Expert Opinion on Biological Therapy 2018;18(12):1257-70.

[6] Powers M, Greven M, Kleinman R, et al. Recent advances in the management and understanding of diabetic retinopathy. F1000Research 2017:6:2063.

[7] Song W, Zhu YW. Chinese medicines in diabetic retinopathy therapies. Chinese Journal of Integrative Medicine 2018;1(5):e182658.

[8] Wang T, Deng Y, Chen Y, et al.Disorderedmetabolism and repair mechanism: mitochondria influenced by cationic and neutral nanoparticles. Journal of Biomedical Nanotechnology 2019;15(12):2428-38. 
[9] Formica ML, Gamboa GU, Tártara LI, et al. Triamcinolone acetonide-loaded lipid nanocapsules for ophthalmic applications. International Journal of Pharmaceutics 2020;573:118795.

[10] Li Z, Sreekumar PG, Peddi S, et al. The humanin peptide mediates ELP nanoassembly and protects human retinal pigment epithelial cells from oxidative stress. Nanomedicine. Nanotechnology, Biology and Medicine 2020;24:102111.

[11] Zeng L, Ma W, Shi L, et al. Poly (lactic-co-glycolic acid) nanoparticle-mediated interleukin-12 delivery for the treatment of diabetic retinopathy. International Journal of Nanomedicine 2019;14:6357-69.

[12] Sims LB, Tyo KM, Stocke S, et al. Surface-modified melphalan nanoparticles for intravitreal chemotherapy of retinoblastoma. Investigative Ophthalmology \& Visual Science 2019;60(5):1696-705.

[13] Zhang XP, Sun JG, Yao J, et al. Effect of nanoencapsulation using poly (lactide-co-glycolide)(PLGA) on antiangiogenic activity of bevacizumab for ocular angiogenesis therapy. Biomedicine \& Pharmacotherapy 2018;107:1056-63.

[14] Shoval A, Markus A, Zhou Z, et al. Anti - VEGF - aptamer modified C - dots - a hybrid nanocomposite for topical treatment of ocular vascular disorders. Small 2019;15(40):1902776.

[15] Narvekar P, Bhatt P, Fnu G, et al.Axitinib-loaded poly (Lactic-Co-Glycolic Acid) nanoparticles for age-related macular degeneration: formulation development and in vitro characterization. Assay and Drug Development Technologies 2019;17(4):167-77.

[16] Halasz K, Kelly SJ, Iqbal MT, et al. Utilization of apatinibloaded nanoparticles for the treatment of ocular neovascularization. Current Drug Delivery 2019;16(2):153-63.

[17] Jeong JH, Nguyen HK, Lee JE, et al. Therapeutic effect of apatinib-loaded nanoparticles on diabetes-induced retinal vascular leakage. International Journal of Nanomedicine 2016;11:3101-9.

[18] Xu L, Li W, Shi Q, et al. Synthesis of mulberry leaf extract mediated gold nanoparticles and their ameliorative effect on Aluminium intoxicated and diabetic retinopathy in rats during perinatal life. Journal of Photochemistry and Photobiology B: Biology 2019;196:111502.

[19] Dong Y, Wan G, Yan P, et al. Fabrication of resveratrol coated gold nanoparticles and investigation of their effect on diabetic retinopathy in streptozotocin induced diabetic rats. Journal of Photochemistry and Photobiology B: Biology 2019;195:51-7.

[20] Qiu F, Meng T, Chen Q, et al. Fenofibrate-loaded biodegradable nanoparticles for the treatment of experimental diabetic retinopathy and neovascular agerelated macular degeneration. Molecular Pharmaceutics 2019;16(5):1958-70.

[21] Kelly SJ, Halasz K, Smalling R, et al.Nanodelivery of doxorubicin for age-related macular degeneration. Drug Development and Industrial Pharmacy 2019;45(5):71523.
[22] Rong X, Ji Y, Zhu X, et al. Neuroprotective effect of insulin-loaded chitosan nanoparticles/PLGA-PEG-PLGA hydrogel on diabetic retinopathy in rats. International Journal of Nanomedicine 2019;14:45-55.

[23] Yang P, Dong Y, Huang D, et al. Silk fibroin nanoparticles for enhanced bio-macromolecule delivery to the retina. Pharmaceutical Development and Technology 2019;24(5):575-83.

[24] Davis BM, Pahlitzsch M, Guo L, et al. Topical curcuminnanocarriers are neuroprotective in eye disease. Scientific Reports 2018;8(1):11066.

[25] Tang M, Ji X, Xu H, et al.Photostable and biocompatible fluorescent silicon nanoparticles-based theranostic probes for simultaneous imaging and treatment of ocular neovascularization. Analytical Chemistry 2018;90(13):8188-95.

[26] Yan J, Peng X, Cai Y, et al. Development of facile drug delivery platform of ranibizumab fabricated PLGAPEGylated magnetic nanoparticles for age-related macular degeneration therapy. Journal of Photochemistry and Photobiology B 2018;183:133-6.

[27] Puglia C, Blasi P, Ostacolo C, et al. Innovative nanoparticles enhance $\mathrm{N}$-palmitoylethanolamide intraocular delivery. Frontiers in Pharmacology 2018;9:285.

[28] Huang D, Chen YS, Rupenthal ID. Hyaluronic acid coated albumin nanoparticles for targeted peptide delivery to the retina. Molecular Pharmaceutics 2017;14(2):533-45.

[29] Deguchi S, Otake H, Nakazawa Y, et al. Ophthalmic formulation containing nilvadipine nanoparticles prevents retinal dysfunction in rats injected with streptozotocin. International Journal of Molecular Sciences 2017;18(12):2720.

[30] Nagai N, Deguchi S, Otake H, et al. Therapeutic effect of cilostazol ophthalmic nanodispersions on retinal dysfunction in streptozotocin-induced diabetic rats. International Journal of Molecular Sciences 2017;18(9):1971.

[31] Wang J, Tan J, Luo J, et al. Enhancement of scutellarin oral delivery efficacy by vitamin B12-modified amphiphilic chitosan derivatives to treat type II diabetes induced-retinopathy. Journal of Nanobiotechnology 2017;15(1):18.

[32] Mitra RN, Nichols CA, Guo J, et al. Nanoparticle-mediated miR200-b delivery for the treatment of diabetic retinopathy. Journal of Controlled Release 2016;236:317.

[33] Zhang P, Liu X, Hu W, et al. Preparation and evaluation of naringenin-loaded sulfobutylether- $\beta$ cyclodextrin/chitosan nanoparticles for ocular drug delivery. Carbohydrate Polymers 2016;149:224-30.

[34] Ren H, Zhu C, Li Z, et al.Emodin-loaded magnesium silicate hollow nanocarriers for anti-angiogenesis treatment through inhibiting VEGF. International Journal of Molecular Sciences 2014;15(9):16936-48.

[35] Mitra RN, Merwin MJ, Han Z, et al. Yttrium oxide nanoparticles prevent photoreceptor death in a lightdamage model of retinal degeneration. Free Radical Biology and Medicine 2014;75:140-8. 